\title{
MiRNA-21 Reverses High Glucose and High Insulin Induced Insulin Resistance in 3T3-L1 Adipocytes through Targeting Phosphatase and Tensin Homologue
}

Authors

Affiliations
H.-y. Ling ${ }^{1,2}$, B. Hu ${ }^{1}$, X.-b. Hü ${ }^{3}$, J. Zhong ${ }^{4}$, S.-d. Feng ${ }^{5}$, L. Qin ${ }^{6}$, G. Liu ${ }^{6}$, G.-b. Wen ${ }^{4}$, D.-f. Liao

Affiliation addresses are listed at the end of the article
Key words

miR-21

- insulin resistance

- PTEN

- AKT

received 20.11.2011

first decision 01.04.2012

accepted $\quad 02.04 .2012$

Bibliography

DOI http://dx.doi.org/

10.1055/s-0032-1311644

Published online:

September 6, 2012

Exp Clin Endocrinol Diabetes

2012; 120: 553-559

(C) J. A. Barth Verlag in

Georg Thieme Verlag KG

Stuttgart · New York

ISSN 0947-7349

\section{Correspondence}

\section{D.-F. Liao}

State Key Laboratory of Chinese Medicine Powder and Medicine Innovation in Hunan (incubation) Hunan University of Chinese Medicine

Changsha 410208 Hunan, China

Tel.: +86/0731/8845 8002

Fax: $+86 / 0731 / 88458111$

dfliao66@yahoo.com.cn

\section{G.-B. Wen}

Institute of Clinical Research/

First Affiliated Hospital

University of South China

Hengyang

China

Tel.: +86/0734/8281340

Fax: $+86 / 0734 / 8281340$

linghongyan@126.com

\section{Abstract}

V

Aims/hypothesis: Our previous study showed there was a change of microRNA (miRNA) expression profile, and miR-21 was significantly down regulated in insulin-resistant adipocytes (IR-adipocytes). Phosphatase and tensin homologs deleted on chromosome 10 (PTEN), a negative regulator of the phosphatidylinositol 3-kinase (PI3K)/AKT pathway, was identified to be a target gene of miR-21, which suggested miR-21 might be associated with insulin resistance (IR) or diabetes. However, it is not known whether miR-21 play any role in the development of IR in 3T3-L1 adipocytes.

Methods: Normal adipocytes and adipocytes transfected with pre-miR-21(pmiR-21) or negative control ( $\mathrm{pNeg}$ ) were treated with high glucose and high insulin for $24 \mathrm{~h}$, insulin-stimulated glucose uptake was determined by 2-Deoxyglucose transport assay, miR-21 expression level was measured by using quantitative real-time

\section{Introduction}

$\nabla$

miRNAs are a novel class of highly conserved, non-coding small RNAs that negatively regulate gene expression via degradation or translational repression of their target mRNAs [1,2]. It has been proved that miRNAs play important roles in energy homeostasis [3], sugar and lipid metabolism [4], insulin secretion [5], pancreatic $\beta$-cell development [6], and adipocyte differentiation [7]. Recent findings showed that some miRNA played a role in the formation of IR. For instance, miR-320 was up-regulated in IR adipocytes, and antisense oligonucleotides against miR-320 ameliorated IR [8]. Over-expression of miR-29 aggravated IR [9]. However, it is unknown whether miR-21 plays a role in the development of IR. Recently, Kim et al. reported miR-21 regu-
RT-PCR (qRT-PCR). The protein expression levels of PTEN, Akt, phospho-Akt (Ser473), IR $\beta$, GSK3ß, phospho-GSK3 $\beta$ (Ser9) and GLUT4 were detected by western blotting assay.

Results: We further confirmed that miR-21 was down regulated in IR-adipocytes by qRT-PCR. Over-expression of miR-21 significantly increased insulin-induced glucose uptake and decreased PTEN protein expression, while it had no significant effect on PTEN mRNA expression in IR-adipocytes. Moreover, over-expressing miR-21 significantly increased insulin-induced phosphorylation of AKT (Ser473), GSK3 $\beta$ (Ser9) and the translocation of glucose transporter 4 (GLUT4) in IR-adipocytes.

Conclusions: In this study, our data demonstrate that miR-21 reverses high glucose and high insulin induced IR in 3T3-L1 adipocytes, possibly through modulating the PTEN-AKT pathway, and miR-21 may be a new therapeutic target for metabolic diseases such as T2DM and obesity.

lated adipogenic differentiation through the modulation of TGF- $\beta$ signaling[10], Dey et al. identified miR-21 as the molecular link between high glucose and phosphatase tensin homologue (PTEN) suppression [11], and Zhang et al. found miR-21 protected from mesangial cell proliferation induced by diabetic nephropathy in $\mathrm{db} / \mathrm{db}$ mice via its target gene PTEN [12]. PTEN is a dual-function lipid and phosphoinositide phosphatase that was reported as a direct target of miR-21 in some diseases $[13,14]$. Studies displayed PTEN negatively regulated insulin signaling through the PI3K pathway in 3T3-L1 adipocytes $[15,16]$. Based on these observations, we infer that miR-21 can influence IR via regulation of PTEN pathway.

In this study, we investigated the potential role and mechanism of miR-21 in the formation of IR 
in 3T3-L1 adipocytes. Our data validated that miR-21 markedly down-regulated in IR adipocytes compared with normal adipocytes. Over-expression of miR-21 significantly increased insulin-stimulated glucose uptake in IR-adipocytes. Consistent with its function, over-expression of miR-21 decreased the expression of PTEN, increased insulin-induced phosphorylation of Akt (Ser473) and GSK3 $\beta$ (Ser9), and promoted GLUT4 translocation in IR-adipocytes. Taken together, the current result firstly demonstrated that miR-21 improved IR in 3T3-L1 adipocytes, possibly through modulating PTEN-AKT pathway.

\section{Materials and Methods \\ $\nabla$ \\ Materials}

3T3-L1 pre-adipocytes were purchased from the American Type Culture Collection (Manassas, VA), 3-isobutyl-1-methylxanthine (IBMX), insulin and dexamethasone were obtained Sigma (St. Louis, MO); Lipofectamine 2000, Trizol reagent and SYBR Green I dye were obtained from Invitrogen (Carlsbad CA), pSilencer ${ }^{\mathrm{TM}}$ 3.1-H1 expression vectors were purchased from Ambion (Austin, TX), polyclonal rabbit against PTEN, IR $\beta$, Akt, phospho-Akt (Ser473), GSK3 $\beta$ and phospho-GSK3 $\beta$ (Ser9) antibodies were purchased from Cell Signaling Technology (Beverly, MA, USA); polyclonal goat anti-GLUT4 and mouse monoclonal anti- $\beta$-actin were purchased from Santa Cruz Biotechnology (Santa Cruz, CA).

\section{Cell culture and induction of insulin resistance}

3T3-L1 pre-adipocytes were propagated and induced to differentiation as described previously [17]. Typically, mature adipocytes appeared within 9 days of differentiation. To induce IR, 3T3-L1 adipocytes were preincubated for $24 \mathrm{~h}$ at $37^{\circ} \mathrm{C}$ with DMEM (10\% FBS) containing $5 \mathrm{mmol} / \mathrm{L}$ glucose with or without $1 \mu \mathrm{mol} / \mathrm{L}$ insulin, or $25 \mathrm{mmol} / \mathrm{L}$ glucose with or without $1 \mu \mathrm{mol} / \mathrm{L}$ insulin.

MiR-21 plasmid construction and transient transfection Oligonucleotides corresponding to the murine precursor sequence of miR-21 were introduced into pSilencer ${ }^{\mathrm{TM}}$ 3.1-H1 vector to obtain pmiR-21 plasmid. The introduced sequences were as follows: sense 5'-GATCCTAGCTTATCAGACT GATG TTGATTCAAGAGATCAACATCAGTCTGATAAGCTATTTTTGGAAA-3'; and antisense 5'-AGCTTTTCCAAAAATCAACATCAGT CTGATAAGCTATCTCTTGAATAGCTTATCAGACTGATGTTGAG-3'. pmiR-21 plasmid was confirmed by DNA sequencing. Empty plasmid pSilencer ${ }^{\mathrm{TM}}$ 3.1-H1 was used as a negative control (pNeg). For transient transfection, mature adipocytes in six-well plates were transfected in triplicate with pmiR-21 or pNeg plasmid using Lipofectamine 2000 for $4 \mathrm{~h}$ ( $4 \mu \mathrm{g}$ oligonucleotide was used in each well).

Quantitative real-time PCR (qRT-PCR) analysis for miR21 and PTEN mRNA expression

For analysis of miR-21 and PTEN mRNA expression, the qRT-PCR was performed as described previously [17]. Briefly, miRNAenriched total RNA was extracted from cells using an RNeasy mini kit, and miR-21 expression was determined using a TaqMan MicroRNA Assay kit (ABI, USA) according to the manufacturer's instruction. The highly conserved snRNA U6 was used as an internal normalizing control. For analysis of PTEN mRNA expression, total RNA was extracted and the mRNA was quantified using SYBR green PCR master mix and a LightCycler Real Time PCR system (Bio-Rad, Hercules, CA, USA). The sequences of PCR the primers were as follows: (i) PTEN forward 5'-CGGCAGCATCAAATGTTT CAG-3' and reverse 5'-AACTGGCAGGTAGAAG GCAACTC-3' and (ii) $\beta$-actin forward $5^{\prime}$-TGTCCACCTTCCAGCAGATGT-3' and reverse 5'-AGCTCAGTAACA GTCCGCCTAGA-3'. The relative expression ratio of miR-21 and PTEN were calculated using the $2^{-\Delta \Delta C t}$ method [18].

\section{2-Deoxyglucose transport assay}

Adipocytes in six-well plates were incubated in serum-free medium for $2 \mathrm{~h}$. Then, cells were washed 3 times with KrebsRinger phosphate buffer (KRPB, consisting of $128 \mathrm{mmol} / \mathrm{L} \mathrm{NaCl}$, $4.7 \mathrm{mmol} / \mathrm{L} \quad \mathrm{KCl}, 5 \mathrm{mmol} / \mathrm{L} \quad \mathrm{NaH}_{2} \mathrm{PO}_{4}, 5 \mathrm{mmol} / \mathrm{L} \quad \mathrm{Na}_{2} \mathrm{HPO}_{4}$, $1.25 \mathrm{mmol} / \mathrm{L} \mathrm{MgSO}_{4}, 1.25 \mathrm{mmol} / \mathrm{L} \mathrm{CaCl}_{2}, \mathrm{pH}$ 7.4) before being incubated for $30 \mathrm{~min}$ at $37^{\circ} \mathrm{C}$ in the presence or absence of $100 \mathrm{nmol} / \mathrm{L}$ insulin. $\left[{ }^{3} \mathrm{H}\right]-2-$ Deoxyglucose $(50 \mu \mathrm{mol} / \mathrm{L} ; 9.25 \mathrm{KBq})$ and 2-deoxyglucose (final concentration $0.1 \mathrm{mmol} / \mathrm{L}$ ) were added to each well for $10 \mathrm{~min}$ and cells were then washed quickly in ice-cold PBS. 2-Deoxyglucose uptake was assayed by scintillation counting. At the same time, ice-cold containing PBS $10 \mu \mathrm{mol} / \mathrm{L}$ containing cytochalasin B was added to each well and the cells were washed 3 times with ice-cold PBS for the measurement of nonspecific uptake. Specific uptake, nonspecific uptake subtracted from total uptake, was determined.

\section{Bioinformatics analysis}

3 programs, miRanda, TargetScan and PicTar, were used to predict the targets of miR-21.

\section{Western blot analysis of total cellular lysates}

Cells were washed twice in ice-cold PBS, and lysed in a buffer containing $10 \mathrm{mmol} / \mathrm{L}$ HEPES (pH7.9), $5 \mathrm{mmol} / \mathrm{L} \quad \mathrm{MgCl}_{2}$, $10 \mathrm{mmol} / \mathrm{L} \mathrm{KCl}$ and $0.5 \% \mathrm{NP}-40$. Cell lysates were collected by centrifuging at $13000 \mathrm{~g}$ for $15 \mathrm{~min}$ at $4^{\circ} \mathrm{C}$. Protein concentrations in the cell lysates were determined by BCA assay. Briefly, sample proteins $(30-50 \mu \mathrm{g})$ were separated by $10 \%$ sodium dodecyl sulphate-polyacrylamide gel electrophoresis (SDS-PAGE) and then transferred to PVDF membranes. The membranes were blocked for $60 \mathrm{~min}$ in a buffer containing $0.1 \%$ Tween-20 and $5 \%$ milk. Antibodies against PTEN, Akt, phospho-Akt (Ser473), IR $\beta$, GSK3 $\beta$, phospho-GSK3 $\beta$ (Ser9) and GLUT4 were used to identify specific proteins, which were then visualized by the ECL method. The intensity of a protein band of interest was quantified by densitometry.

\section{Western blot analysis of total cellular membrane GLUT4 [19]}

Cells were washed twice in ice-cold HES buffer $(20 \mathrm{mmol} / \mathrm{L}$ HEPES, pH 7.5; $1 \mathrm{mmol} / \mathrm{L}$ EDTA; and $250 \mathrm{mmol} / \mathrm{L}$ sucrose) and scraped in HES buffer in the presence of protease inhibitors. Samples were homogenized (30 strokes in a glass Dounce homogenizer) at $4{ }^{\circ} \mathrm{C}$ and centrifuged at $1000 \times \mathrm{g}$ for $5 \mathrm{~min}$ to remove unbroken cells, supernatant was further centrifuged at $16000 \times \mathrm{g}$ for $15 \mathrm{~min}$ to produce a crude plasma membrane fraction. The resulting pellet was resuspended in a $1.15 \mathrm{~mol} / \mathrm{L}$ sucrose cushion (HES buffer containing $1.15 \mathrm{~mol} / \mathrm{L}$ sucrose), and centrifuged at $100000 \times \mathrm{g}$ in swing rotor for $60 \mathrm{~min}$. The white fluffy band at the interface was collected, diluted in HES, and centrifuged again at $48000 \times \mathrm{g}$ for $60 \mathrm{~min}$, yielding a pellet of the 
plasma membrane. The amount of GLUT4 in the plasma membrane was determined by immunoblotting using anti-GLUT4 antibody.

\section{Statistical analysis}

Data are presented as the mean \pm SD. 2 groups were compared by unpaired Student's $t$ test and multiple groups were analyzed by one-way analysis of variance (ANOVA) followed by Dunnett's test. $P<0.05$ was considered significant.

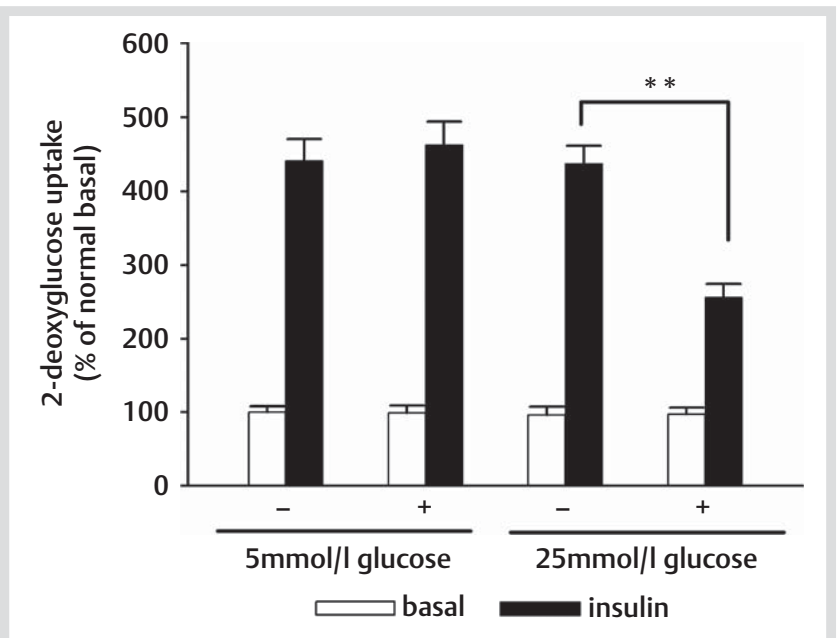

Fig. 1 The establishment of insulin-resistant adipocytes. 3T3-L1 adipocytes were preincubated for $24 \mathrm{~h}$ in media containing $5 \mathrm{mmol} / \mathrm{L}$ glucose or $25 \mathrm{mmol} / \mathrm{L}$ glucose with (+) or without (-) $1 \mu \mathrm{mol} / \mathrm{L}$ insulin, respectively. Cells were washed and acutely stimulated with or without $100 \mathrm{nmol} / \mathrm{L}$ insulin for $30 \mathrm{~min}$. 2-DG uptake was measured as described in methods. The degree of basal glucose uptake in adipocytes treated with $5 \mathrm{mmol} / \mathrm{L}$ glucose (normal adipocytes) was set to $100 \%$. Data are the mean $\pm S D(n=3):{ }^{* *} P<0.01$.

\section{Results}

$\nabla$

\section{Establishment of insulin-resistant adipocytes}

To evaluate the insulin-resistant 3T3-L1 adipocyte model, 2-deoxyglucose (2-DG) uptake was measured by radioimmunoassay to determine the insulin sensitivity of the cells. As expected after differentiation, 3T3-L1 adipocytes exhibited a marked response to an acute maximal dose of insulin $(100 \mathrm{nmol} / \mathrm{L})$ with significant increases in glucose transport above basal under all conditions studied. When cells were preincubated for $24 \mathrm{~h}$ in media containing $5 \mathrm{mmol} / \mathrm{L}$ glucose (normal adipocytes), acute insulin stimulation $(100 \mathrm{nmol} / \mathrm{L})$ induced a 4.4-fold increase in 2-DG transport. The addition of $1 \mu \mathrm{mol} / \mathrm{L}$ insulin during preincubation did not significantly affect either basal or insulin-stimulated 2-DG transport. Also, when cells were preincubated without insulin in $25 \mathrm{mmol} / \mathrm{L}$ glucose, the acute insulin response of glucose transport was not significantly different when compared with cells preincubated in $5 \mathrm{mmol} / \mathrm{L}$ glucose. However, $24 \mathrm{~h}$ of preincubation in media containing high glucose in the presence of $1 \mu \mathrm{mol} / \mathrm{L}$ insulin decreased acute insulin-stimulated glucose transport by $41.4 \%$, when compared with cells preincubated with $25 \mathrm{mmol} / \mathrm{L}$ glucose. The data indicated that treatment of 3T3-L1 adipocytes with high glucose $(25 \mathrm{mmol} / \mathrm{L})$ and high insulin $(1 \mu \mathrm{mol} / \mathrm{L})$ resulted in the induction of IR ( $\odot$ Fig. 1 ).

\section{Reduced expression of miR-21 in insulin-resistant adipocytes}

We have previously shown that there was a differential expression profile of miRNAs between normal adipocytes (control group) and insulin-resistant adipocytes (IR-adipocytes) (IR group). Among them, miR-21 was the most significantly downregulated miRNA, which was 16.5 -fold reduction in the IR group compared with control group [8]. To further confirm miR-21 expression, qRT-PCR analysis was used. As shown in 0 Fig. 2a, miR-21 was significantly decreased (about 5.3 folds) in the IR

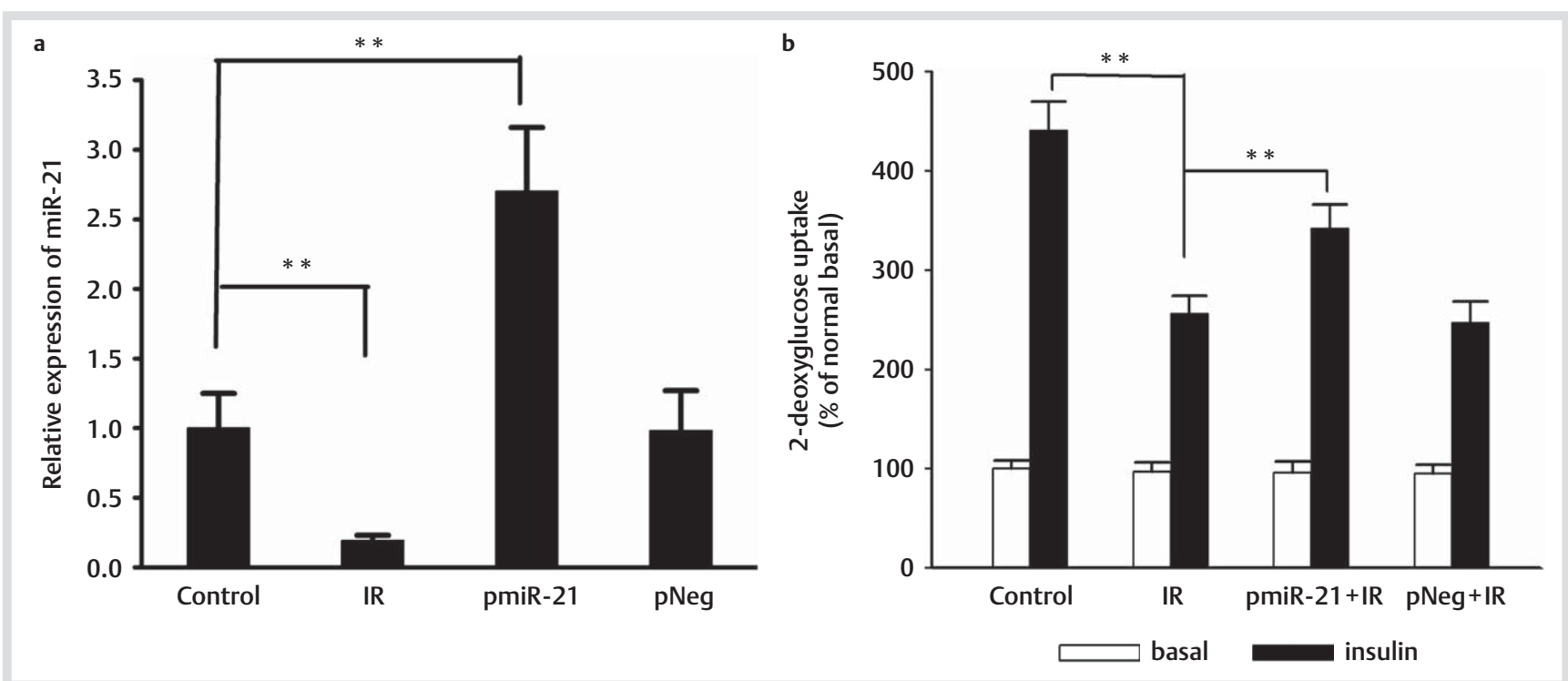

Fig. 2 Over-expression of miR-21 increased insulin-induced glucose uptake in IR-adipocytes. Normal adipocytes (control), miR-21-transfected adipocytes (pmiR-21) and empty plasmid pSilencer ${ }^{\mathrm{TM}}$ 3.1- $\mathrm{H} 1$-transfected adipocytes ( $\mathrm{pNeg}$ ) were induced to insulin-resistant adipocytes with high glucose and high insulin for $24 \mathrm{~h}$ as the IR group, pmiR-21+IR group and pNeg+IR group, respectively. a Relative expression ratio of miR-21 was measured by quantitative real-time reverse transcription polymerase chain reaction (qRT-PCR). The expression levels of miR-21 in the Control group were considered as 1 , its relative expression levels in other groups were presented as a ratio with that of Control group. $\mathbf{b}$ Insulin-induced glucose uptake was determined by 2-Deoxyglucose transport assay. The degree of basal glucose uptake in control group was set to $100 \%$. Data are the mean $\pm \operatorname{SD}(n=3) .{ }^{* *} p<0.01$. 
mmu PTEN 3'UTR

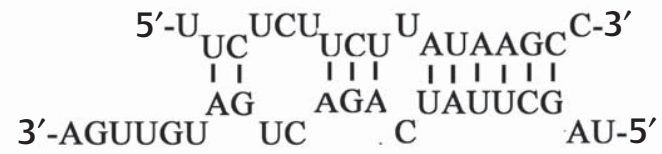

mmu PTEN 3'UTR

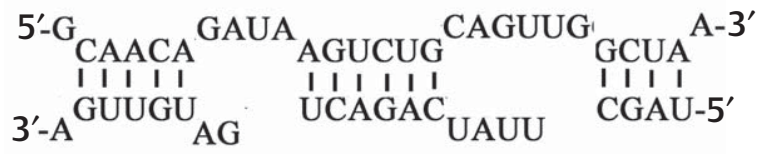

miR-21

UAUU

Fig. 3 PTEN is a target of miR-21 in 3T3-L1 adipocytes. 3T3-L1 adipocytes of control, pmiR-21 and pNeg were induced to IR-adipocytes with high glucose and high insulin for $24 \mathrm{~h}$ as the IR group, pmiR-21+IR group and pNeg+IR group, respectively. a Sequence complementarity between miR-21 and its target sites in the 3' UTR of human (hsa), mouse (mmu) and rat (rno) PTEN mRNAs. b PTEN protein levels were measured by Western blot analysis. Representative immunoblots (top) and densitometric analysis (bottom) on the PTEN protein levels normalized to internal $\beta$-actin level were reported. c PTEN mRNA expression was measured using qRT-PCR, relative mRNA levels were calculated as relative change from the Control group level. Data are the mean \pm SD $(n=3)$. ${ }^{*} P<0.01$.

group compared to control group, which was consistent with the microarray data. Together, it suggested that miR-21 expression was inversely related to IR.

\section{Over-expression of miR-21 increased insulin-induced glucose uptake in IR-adipocytes}

Results of qRT-PCR showed that miR-21 was significantly increased in the miR-21-transfected adipocytes (pmiR-21) compared with empty plasmid pSilencer ${ }^{\mathrm{TM}}$ 3.1-H1-transfected adipocytes (pNeg), suggesting miR-21 can be over-expressed by
Lipofectamine 2000 transfection in 3T3-L1 adipocytes ( $\bullet$ Fig. 2a). To investigate the impact of miR-21 on the formation of IR, glucose uptake was determined after pmiR-21 or pNeg were treated with high glucose and high insulin for $24 \mathrm{~h}$. As shown in $\odot$ Fig. $\mathbf{2 b}$, in the absence of insulin stimulation, there was no significant difference in glucose uptake in all groups. However, following insulin stimulation, IR adipocytes exhibited a marked reduction in insulin-induced glucose uptake (an approximate 1.7-fold reduction in the absence or presence of pNeg treatment), which was restored (46.5\% recovery) following the treatment of cells 

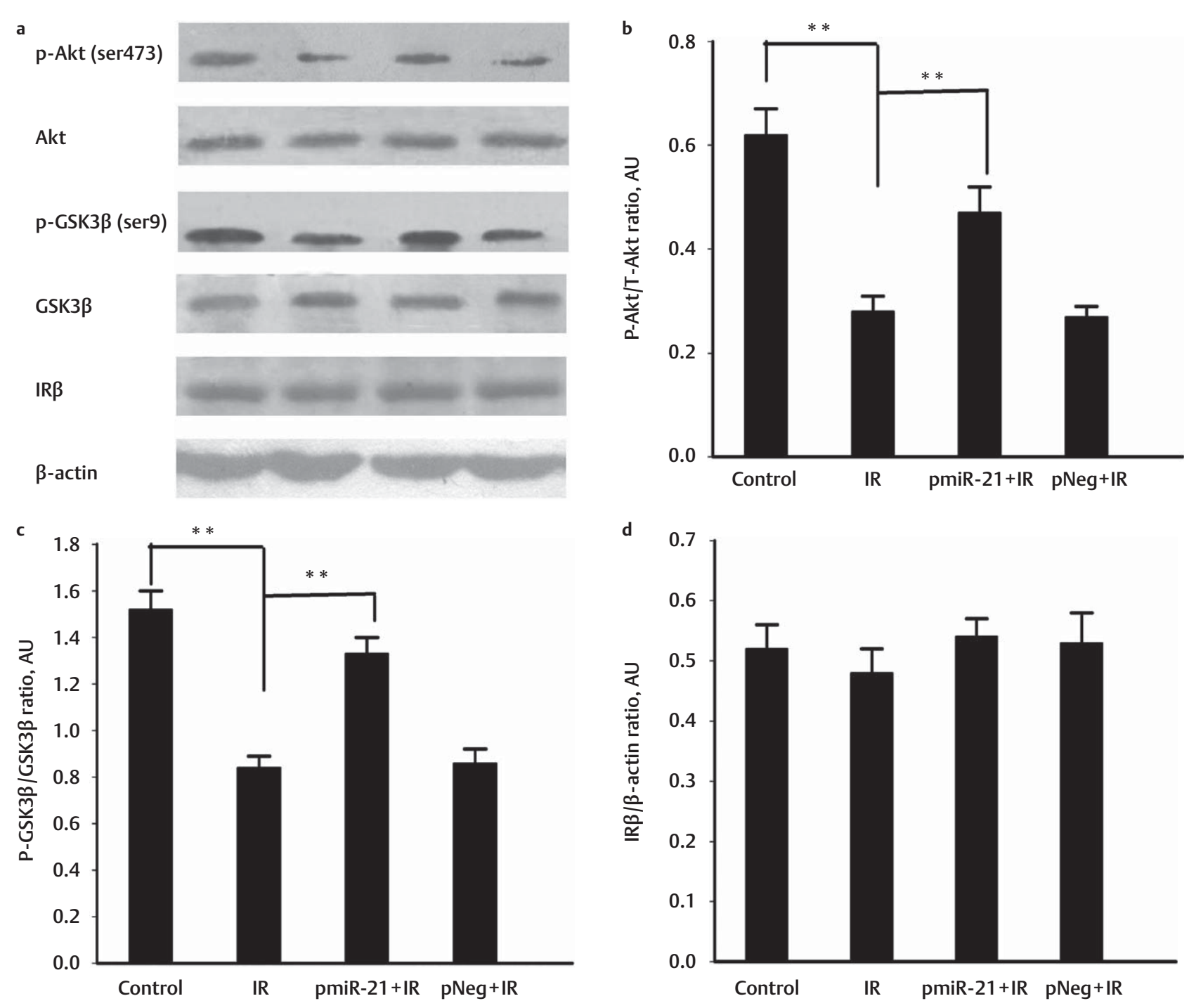

Fig. 4 Over-expression of miR-21 led to increased Akt signaling in IR-adipocytes. 3T3-L1 adipocytes of control, pmiR-21 and pNeg were induced to IRadipocytes with high glucose and high insulin for $24 \mathrm{~h}$ as the IR group, pmiR-21+IR group and pNeg+IR group, respectively. Cell lysates were collected and subjected to western blot analysis using antibodies specific for P-Akt, P-GSK3 $\beta$ and IR $\beta$ a. Densitometry data of Akt phosphorylation, GSK3 $\beta$ phosphorylation and IR $\beta$ protein levels in 3T3-L1 cells were showed, respectively $\mathbf{b}, \mathbf{c}$ and $\mathbf{d}$. Data are expressed as the mean $\pm \operatorname{SD}(n=3) .{ }^{*} P<0.01$.

with pmiR-21. The data indicated that a negative role of miR-21 in the regulation of IR, over-expression of miR-21 significantly promoted insulin-induced glucose uptake in IR-adipocytes.

\section{PTEN is a target of miR-21 in 3T3-L1 adipocytes}

PTEN has been reported as a direct target of miR-21 in some diseases $[13,14]$. Recent studies demonstrate that nearly $25 \%$ of miRNA target sites in the 3'UTR are conserved in humans and mice. Analysis of human, mouse and rat PTEN 3'UTR showed the presence of the highly conserved miR-21 recognition element ( $\bullet$ Fig. 3a). Therefore we predicted PTEN might be a potential target gene of miR-21 in 3T3-L1 adipocytes. To confirm this hypothesis, we examined the mRNA and protein expression of PTEN by qRT-PCR and Western blot analysis, respectively. Our data showed PTEN mRNA and protein levels were significantly increased in IR adipocytes compared with normal adipocytes, over-expression of miR-21 significantly decreased PTEN protein level, whereas it had no significant effect on PTEN mRNA expres- sion in IR-adipocytes. These data indicated that PTEN was a target gene of miR-21 in 3T3-L1 adipocytes ( $\bullet$ Fig. 3b, c).

\section{Over-expression of miR-21 led to increased insulin signaling in IR-adipocytes}

PTEN is a negative regulator of PI3K-dependent signaling. Activation of PI3-K results in the activation of Akt and downstream mediators involved in insulin signaling such as GSK3. To investigate the potential involvement of PTEN in the regulation of insulin signaling by miR-21, Akt phosphorylation at Ser474 (Akt-PSer474) was determined by Western blot analysis. As shown in $\bullet$ Fig. 4, IR-adipocytes exhibited a significant $54.8 \%$ reduction in insulin-stimulated Akt-PSer474 compared with normal adipocytes. This decrement was significantly recovered $(58.9 \%$ recovery) by treatment of IR-adipocytes with pmiR-21. To further explore whether the Akt pathway is activated, we determined the level of phospho-GSK3 $\beta$ (Ser9), a substrate of Akt kinase activity in adipocytes. There were about 1.8 -fold decreases of phospho-GSK3 $\beta$ protein level in IR-adipocytes com- 


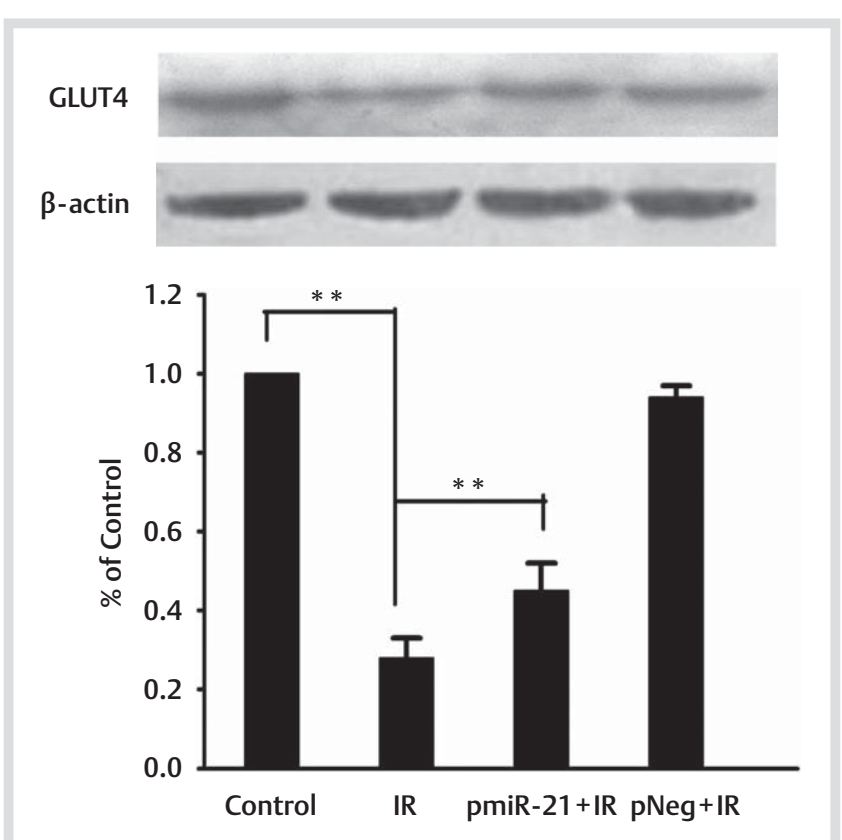

Fig. 5 Over-expression of miR-21 promoted insulin-stimulated GLUT4 translocation in IR-adipocytes. 3T3-L1 adipocytes of control, pmiR-21 and pNeg were induced to IR-adipocytes with high glucose and high insulin for $24 \mathrm{~h}$ as the IR group, pmiR-21+IR group and pNeg+IR group, respectively. The plasma membrane of 3T3-L1 cells was fractionated, and the GLUT4 protein level was determined by Western blot analysis. Representative immunoblots and densitometric analysis were reported. The amounts of GLUT4 were expressed as percentage of that in the Control group. Data are expressed as the mean \pm SD $(n=3)$. ${ }^{*} P<0.01$.

pared to normal adipocytes. Furthermore, IR-adipocytes overexpressing miR-21 resulted in an increase in insulin-induced phosphorylation of GSK3 $\beta$ by $~ 1.6$-fold, relative to untreated or pNeg-treated IR-adipocytes, whereas the Akt protein levels remained the same. No difference was observed with PTEN upstream signaling molecules such as IR- $\beta$ ( $\bullet$ Fig. 4). Taken together, these results suggested the inhibition of PTEN expression by miR-21 led to activation of the PI3K/Akt pathway without perturbing PTEN upstream signaling molecules.

\section{Over-expression of miR-21 promoted insulin- stimulated GLUT4 translocation in IR-adipocytes}

Akt, a serine/threonine protein kinase, is one of the downstream target molecules of PI3K and has been shown to mediate the metabolic actions of insulin. Activated Akt plays a significant role in promoting GLUT4 translocation. To assess GLUT4 translocation, we performed SDS-PAGE and Western blot analysis using anti-GLUT4 antibody in total cell lysates and plasma membrane lawns ( $\odot$ Fig. 5). We found that high glucose and high insulin decreased insulin-stimulated GLUT4 translocation to plasma membrane by $61.7 \%$, over-expression of miR- 21 exhibited a significant $36.9 \%$ increase in GLUT4 protein level compared with untreated or pNeg-treated IR-adipocytes, which agreed well with the glucose uptake results. However, over-expression of miR-21 had no significant effect on total GLUT4 protein expression in IR-adipocytes (data not shown). Taken together, these observations implicated that over-expression of miR-21 reversed insulin resistance in 3T3-L1 adipocytes through PTEN-Akt pathway.

\section{Discussion}

$\nabla$

Insulin resistance (IR) is the pathogenic hallmark of type 2 diabetes mellitus (T2DM). Therefore, further exploration into the molecular mechanism of IR will contribute to the prevention and treatment for T2DM and its complications. Emerging evidence suggests that miRNAs play an important role in diabetes and its related complications [12,20,21]. Some studies showed miR-21 might be associated with IR or diabetes [10-12]. However, the role of miR-21 in the development of IR was not clear. In the current study, we further confirmed that the expression of miR-21 was reduced, and miR-21 significantly increased insulinstimulated glucose uptake in IR-adipocytes ( $\bullet$ Fig. 2 ), suggesting that miR-21 enhanced insulin sensitivity and improved IR. What are the underlying mechanisms that miR-21 improved IR? Physiologically, insulin signals passes through a pathway involving protein kinases including, but not limited to, PI3K, AKT or protein kinase B (PKB), and GSK-3 $\beta$ (the PI3K/AKT/GSK-3 $\beta$ pathway) [22]. Emerging evidence suggests that IR can potentially be treated via modulation of the PI3K/AKT pathway by targeting its up- or downstream modulators [23]. Studies also indicated PTEN could inhibit insulin signaling and antagonized PI3Kmediated signaling, and the inhibition of PTEN may enhance insulin signaling $[15,16]$. Our data show that PTEN may be a target of miR-21 and miR-21 negatively regulated the process of IR by targeting PTEN. First, a search with Targetscan revealed that miR-21 is complementary to sites in the 3 ' untranslated regions (3'UTR) of PTEN ( $\bullet$ Fig. 3a). Second, the induction of IR significantly increased PTEN protein expression, which occurred concomitant with a 5.3-fold reduction in miR-21 expression. Third, the enhancement in PTEN protein expression was recovered following treatment of IR-adipocytes with miR-21, while PTEN mRNA expression was not changed.

AKT is a downstream signal molecule of PTEN and AKT activation is a hallmark of PTEN loss [24]. To further confirm the involvement of PTEN in miR-21-mediated effects, Akt phosphorylation was determined. Our data showed that endogenous Akt protein level did not significantly change, while the phosphorylation level of Akt significantly decreased in the IR group compared with control group. Over-expression of miR-21 resulted in an increase in Akt phosphorylation and a concomitant reduction in PTEN expression ( $\bullet$ Fig. 4), Similar results were obtained concerning GSK3 $\beta$ (Ser9) phosphorylation level. GSK3 $\beta$, a substrate of Akt kinase activity, plays an important role in the regulation of glycogen synthesis. Phosphorylation of Ser 9 in GSK3 $\beta$ leads to the inhibition of its activity, so, miR-21 may inhibit GSK-3 $\beta$ through the phosphorylation. Because the limit of experiment condition, we did not detect the PI3K activity, but we observed there was no difference that PTEN upstream signaling molecules such as IR- $\beta$ ( $\bullet$ Fig. 4). Taken together, these data demonstrated the inhibition of PTEN expression led to activation of AKT-GSK3 $\beta$ signal pathway without perturbing PTEN upstream signaling molecules.

Akt is required for GLUT4 translocation to the cell surface following insulin stimulation, which subsequently augments glucose transport [25]. We found insulin-stimulated glucose uptake and GLUT4 translocation were significantly decreased in IR-adiocytes, and miR-21 reversed these changes ( $\bullet$ Fig. 4,5 ). These results reinforce the notion that miR-21 improved IR by PTENAKT signaling, miR-21 could be a causal factor of the down-regulation of PTEN and activation of PI3K/AKT pathway in IR-adipocytes. 
In conclusion, our study indicated that miR-21 reversed high glucose and high insulin induced IR in 3T3-L1 adipocytes through modulating the PTEN-AKT pathway, and miR-21 might be a novel potential target for prevention and therapy of IR and other metabolic diseases.

\section{Acknowledgements}

The work was supported by grants from the National Natural Science Foundation of China (81000328, 81000946, 30971170), the Construct Program of the Key Discipline in Hunan Province and the Constructing Program of Key Disciplines in Hunan Province. This work was supported by the fifty-first batch of China Postdoctoral Sciences Foundation (No. 2012M511384).

\section{Conflict of Interest: None.}

\section{Affiliations}

${ }^{1}$ Department of Physiology, School of Medicine, University of South China, Hengyang, China

${ }^{2}$ Center for basic medical post-doctoral studies, University of South China, Hengyang, China

${ }^{3}$ Department of Biochemistry and Molecular Biology, School of life sciences and Technology, University of South China, Hengyang, China

${ }^{4}$ Institute of Clinical Research/First Affiliated Hospital, University of South China, Hengyang, China

${ }^{5}$ Department of Epidemiology, School of Public Health, University of South China, Hengyang, China

${ }^{6}$ Key Laboratory for Pharmacoproteomics of Hunan Province/Institute of Pharmacy and Pharmacology, University of South China, Hengyang, China ${ }^{7}$ Division of Stem Cell Regulation and Application, State Key Laboratory of Chinese Medicine Powder and Medicine Innovation in Hunan (incubation), Hunan University of Chinese Medicine, Changsha Hunan, China.

\section{References}

1 Bartel DP. MicroRNAs:genomics, biogenesis, mechanism, and function. Cell 2004; 116 (2): 281-297

2 Ambros $V$. The functions of animal microRNAs. Nature 2004; 431 (7006): 350-355

3 Teleman AA, Cohen SM. Drosophila lacking microRNA miR-278 are defective in energy homeostasis. Genes \& Dev 2006; 20 (11): 417-422

4 Poy MN, Spranger M, Stoffel M. MicroRNAs and the regulation of glucose and lipid metabolism. Diabetes Obes Metab 2007; 9 (Suppl 2): $67-73$

5 Poy MN, Eliasson L, Krutzfeldt J et al. A pancreatic islet-specific microRNA regulates insulin secretion. Nature 2004; 432 (7014): 226-230

6 Kloosterman WP, Lagendijk AK, Ketting RF et al. Targeted inhibition of miRNA maturation with morpholinos reveals a role for miR-375 in pancreatic islet development. PLoS Biol 2007; 5 (8): e203

7 Sun TW, Fu MG, Bookout AL et al. MicroRNA let-7 regulates 3T3-L1 adipogenesis. Mol Endocrinol 2009; 23 (6): 925-931

8 Ling HY, Ou HS, Feng SD et al. Changes in microRNA profile and effects of miR-320 in insulin-resistant 3T3-L1 adipocytes. Clin Exp Pharmacol Physiol 2009; 38: e32-e39
9 He A, Zhu L, Gupta N et al. Overexpression of micro ribonucleic acid 29 , highly up-regulated in diabetic rats, leads to insulin resistance in 3T3-L1 adipocytes. Mol Endocrinol 2007; 21 (11): 2785-2794

10 Kim YJ, Hwang SJ, Bae YC et al. MiR-21 Regulates Adipogenic Differentiation through the Modulation of TGF-b Signaling in Mesenchymal Stem Cells Derived from Human Adipose Tissue. Stem Cells 2009; 27 (12): 3093-3102

11 Dey N, Das F, Mariappanl MM et al. icroRNA-21 orchestrates high glucose-induced signals to TORC1 for renal cell pathology in diabetes. J Biol Chem 2011; 286 (6): 25586-25603

12 Zhang $Z$, Peng $H$, Chen $J$ et al. MicroRNA-21 protects from mesangial cell proliferation induced by diabetic nephropathy in $\mathrm{db} / \mathrm{db}$ mice. FEBS Letters 2009; 583 (45): 2009-2014

13 Roy S, Khanna S, Hussain SR et al. MicroRNA expression in response to murine myocardial infarction: miR-21 regulates fibroblast metalloprotease-2 via phosphatase, tensin homologue. Cardiovasc Res 2009; 82 (1): $21-29$

14 Iliopoulos D, Jaeger SA, Hirsch HA et al. STAT3 activation of miR-21 and miR-181b-1 via PTEN and CYLD are part of the epigenetic switch linking inflammation to cancer. Mol Cell 2010; 39 (4): 493-506

15 Tang XQ Powelka AM, Soriano NA et al. PTEN, but Not SHIP2, Suppresses Insulin Signaling through the Phosphatidylinositol 3-Kinase/ Akt Pathway in 3T3-L1 Adipocytes. J Biol Chem 2005; 280 (23): 22523-22529

16 Nakashima $N$, Sharma PM, Imamura $T$ et al. The tumor suppressor PTEN negatively regulates insulin signaling in 3T3-L1 adipocytes. J Biol Chem 2000; 275 (17): 12889-12895

17 Ling HY, Wen GB, Feng SD et al. MicroRNA-375 promotes 3T3-L1 adipocyte differentiation through modulation of extracellular signalregulated kinase signaling. Clin Exp Pharmacol Physiol 2011; 38 (4): 239-246

18 Livak KJ, Schmittgen TD. Analysis of relative gene expression data using real-time quantitative PCR and the 2[-Delta Delta C(T)] method. Methods 2001; 25 (4): 402-408

19 Perrini S, Natalicchio A, Laviola L et al. Dehydroepiandrosterone stimulates glucose uptake in human and murine adipocytes by inducing GLUT1 and GLUT4 translocation to the plasma membrane. Diabetes 2004; 53 (1): 41-52

20 Xiao J, Luo X, Lin $\mathrm{H}$ et al. MicroRNA miR-133 represses HERG K+ channel expression contributing to QT prolongation in diabetic hearts. J Biol Chem 2007; 282 (17): 12363-12367

21 Kato M, Zhang J, Wang $M$ et al. MicroRNA-192 in diabetic kidney glomeruli and its function in TGF-beta-induced collagen expression via inhibition of E-box repressors. Proc Natl Acad Sci 2007; 104 (9): 3432-3437

22 Taniguchi CM, Emanuelli B, Kahn CR. Critical nodes in signalling pathways: insights into insulin action. Nat Rev Mol Cell Biol 2006; 7 (2): 85-96

23 Jiang G, Zhang BB. Pi3-kinase and its up- and down-stream modulators as potential targets for the treatment of type II diabetes. Front Biosci 2002; 7 (4): d903-d907

24 Shepherd PR, Kahn BB. Glucose transporters and insulin action- implications for insulin resistance and diabetes mellitus. N Engl J Med 1999; 341 (4): 248-257

25 Van $D E$, Govers $R$, James DE. Akt activation is required at a late stage of insulin-induced GLUT4 translocation to the plasma membrane. Mol Endocrinol 2005; 19 (4): 1067-1077 